\title{
On the Construction of Intelligent Diagnostic System Cognitive Images Based
}

\author{
Boris A. Kobrinskii \\ Support Systems Laboratory of clinical decision \\ Institute of Modern Information Technologies in Medicine \\ of the FRC "Computer Science and Control” RAS \\ Moscow, Russia \\ Email: bak@isa.ru
}

\begin{abstract}
In intelligent medical systems traditional logical-andlinguistic models do not provide a comparison between expert cognitive performance and in linguistic form of display holistic visual images of the disease in knowledge base. Possible decision may be to build a hybrids linguistic and image frame or ontological models that integrate are qualitative and visual imaginative indicators for the formal representation of diagnostically important clinical manifestations of disease. In this regard, the questions represent different images as an image visual sequences, options to include them in the knowledge and principles of the hybrid logic-and-linguistic-and-image expert system.
\end{abstract}

Keywords-Knowledge engineering; cognitive imaging; cognitive linguistic-and-imaging models; frame; ontology; visual imaginative indicator; intelligent system; medicine diagnostic; hereditary diseases.

\section{INTRODUCTION}

Expert (intelligent) systems, including health care, are already significant in the history of Russian and world science and practice. In their creation to apply and develop various methods of knowledge representation models and building systems architecture. However, to date, some specific features of the medical domain did not find appropriate reflection in the created expert systems. Intelligent systems based on symbolic component, whereas in the natural intelligence (in medical practice) are of considerable importance image representations.

In 80-90 years of the XX century to decision support in the medical field of congenital and hereditary diseases in different mills it established a number of diagnostic systems, some of which were subsequently modified. Among them are french system GENDIAG [1], australian POSSUM - Pictures Of Standard Syndromes and Undiagnosed Malformations [2], british London Neurology DataBase and London Dysmorphology DataBase [3], russian DIAGEN [4]. In these systems, a variety of approaches have been used to knowledge representation, data analysis and output mechanisms. The first presentation of photos of patients the doctor has been realized, but only after the issuance of the diagnostic sequence. To overcome medical errors in the assessment of phenotypic features of hereditary diseases in [4] selection of a particular trait (eg beaked nose, eagle, plum, pear, etc.). When you enter a specific patient data has been replaced by the more general concept (for example, the unusual shape of the nose). Subsequently, in the process of diagnostic hypotheses on the context of the ongoing refinement of the features of the nose form corresponding to the description of a presumptive diagnosis. This was done by analyzing the various contextrelated options for the form of the nose with the other clinical manifestations in various diseases. But this is not a universal solution. The integrity of this, for example, fuzzy visual image as "coarse facial features," his holism, can not be fully reflected in the knowledge base. This is due to a lack of deep-developed methods and tools for the formal description of the ontological model of medical diagnostics, where in a single formalism would be used verbal and figurative knowledge. However, this approach is particularly important in the diagnosis of rare (orphan) hereditary diseases characterized by specific appearance (phenotype). Presentation of pictures of patients the doctor was very important, but it happened only after the diagnostic sequence hypotheses. Orphan diseases are currently being considered as an important medical and social problem [5].

\section{FORMULATION OF THE PROBLEM}

The aim of the research is to develop methods of representation and processing of knowledge of different modality for use in intelligent medical diagnostic decision support systems. New models and methods of formal specification of medical knowledge based on pseudo-physical logics and linguistic and imaginative approach will be used in building intelligent diagnostic system based on cognitive images.

In the early 21 th century dramatically increased interest in creating photo database of patients for their use in clinical practice. This is because more than 700 hereditary disease lead to specific changes of face shape, size and arrangement of the eyes, ears, nose, lips. In the UK, for the purpose of training physicians to recognize the syndromes on the details or the characteristic of the different hereditary diseases were created models, providing visualization of variations 3D face shapes (three-dimensional facial morphology) [6,7]. Also, as a diagnostic indicator for the recognition of morphological disk disease were asked to use specific skin manifestations [8]. In the United States to assist in the identification of various diseases, including genetic nature, in the University of Miami Miller School of Medicine has been used tool visual diagnostic 
support VisualDx [9]. The database created by the system includes more than 24,000 images [10]. However, the system of a visual representation various features diseases are autonomous tools desicion diagnostic support by viewing images of a disease after a preliminary diagnosis of a doctor posing. They are not included in the traditional computer-assistance systems support diagnostic decisions. Movement in this direction can be seen in the English study [11], unites three approaches: (a) train a computer vision algorithm for automatic annotation of 36 feature points of interest across the face on foto, (b) the use of active probabilistic models of face dysmorphism within the nosological groups in a multidimensional space, (c) machine learning using the nearest neighbor method. Clusters of patients in the space known syndromes facilitate the generation of diagnostic hypotheses. Database collection consists of 2878 images, including 1515 healthy controls and 1363 pictures for eight known developmental disorders. According to the authors of the proposed approach helps to reduce potential diagnoses search space in the 27.6 times when a physician suspected developmental disorders. Computer based diagnostic decision support systems based two (2D) or threedimensional (3D) models of facial morphology, created at Ankara University, are showing potential in syndrome discrimination [12]. It might be helpful to assist medical professionals in genotypephenotype dysmorphic diagnosis on basis the differences between facial regions such as facial landmarks, eyebrows, hair, lips, and chins.

At the same time, a doctor, especially having a pronounced image thinking, in the process of diagnostic hypotheses appear associatively "pop-up" visual images of clinical manifestations of similar diseases, sometimes "pulling" for a more pathological symptoms related to verbal characterized by signs. However, well-developed methods and tools for decision support in medical diagnosis that integrate verbal knowledge and cognitive images are virtually absent at present. Note that the image representations are an important criterion in terms of the diagnostic. In the same time visual images are presented to physicians without decision support, but only in the viewing form after setting preliminary diagnosis. At the same time, it is the union verbal and visual characteristics of the pathological manifestations in intellectual system may help to facilitate and improve the differential diagnosis.

The solution to this problem is possible on the basis of the analysis and formalization of knowledge about the manifestations of the disease with the inclusion of information on relevant for the diagnosis of visual images that characterize the features of the phenotype (appearance of patients with different diagnoses).

\section{FEATURES OF REPRESENTATION OF VISUAL IMAGES}

Images - it are holistic or difficult to dismember "phenomena", in the latter case - with the loss of part of its inner content. Verbal interpretation is not always intact, often uncertainty or difficult comprehensibility, as it does not clearly characterized by semantic representation, leaving the field for a variety of interpretations.

Highly qualified doctors specialized clinics may be experts in the construction of linguistic-imaginative diagnostic systems while using the descriptions and photographs of patients with rare diseases in the scientific literature. To do this, knowledge extraction process to create a specific diagnostic expert system, the information on mental visual images that comes into minds of experts must be extracted along with verbalized presentations; the necessary images of patients, which are concomitant to the described clinical manifestations of a disease and have a differential diagnostic value, must be also obtained from them.

Expert system should take into account the ethnic and individual characteristics and age-related changes of clinical symptoms and the appearance of the patients. It should be noted that most visual images are attended by many similar or nearly-similar images or events that recur in memory. Similar recurrences of the image that comply with one notion will be considered as a set, or a visual sequence. As an example, face series represented by children with Down syndrome, which until recently were considered indistinguishable. But as can be seen from Figure 1, they are distinguishable, but similar.

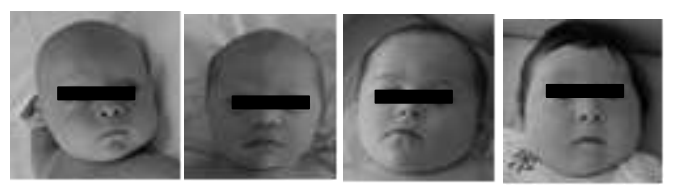

Fig.1. Image series of children with Down syndrome

The fuzziness of transitions between separate representatives of a specific sequence of images permits one to speak about a visual-image quasi-continuum [13, 14]. A temporal (dynamic) image series is characterized by how separate images that form it are transformed or metamorphized in time while maintaining them the general and typical. As a whole, this is a series with the changing features of a face, which is joined by the common idea of the image of a specific person or definite disease. The dynamics of outward appearance are particularly typical for the diseases with a progressive trend. An attempt to verbalize temporally fuzzy transitions destroys the unity of an image. Meanwhile, a visual series demonstrates the unity and simultaneously diversity of images presented in different periods of life. Visual images as part of the traditional knowledge base should be seen as a kind of symbols.

\section{SYSTEM ARCHITECTURE}

The architecture of the hybrid linguistic-and-image the intellectual system is similar to the traditional one. But due to the inclusion image component there is a necessity of its reflection in the knowledge base (Figure 1).

The knowledge base of linguistic type, supplemented by sequences of visual images, transformed into a hybrid knowledge base. In addition, the unit includes a user interaction module reflection visual images.

\section{THE FORMALIZATION OF LINGUISTIC-AND-IMAGE MODELS}

As part of the above stated problems of the challenge is to develop new linguistic and imaginative models that integrate quantitative and qualitative indicators in order to create adequate methods and means of computer medical diagnostics. As a means of formal description of medical knowledge can be viewed frame-based or ontological model. Principle of 
building a hybrid (linguistic and image-bearing) frame or ontological models that integrate both qualitative and visual image indicators aimed at the formal description of the disease clinical manifestations that have a diagnostic value are reflected in a number of publications [13, 14]. Ontology and frame fragments are shown in Figure 2 and 3.

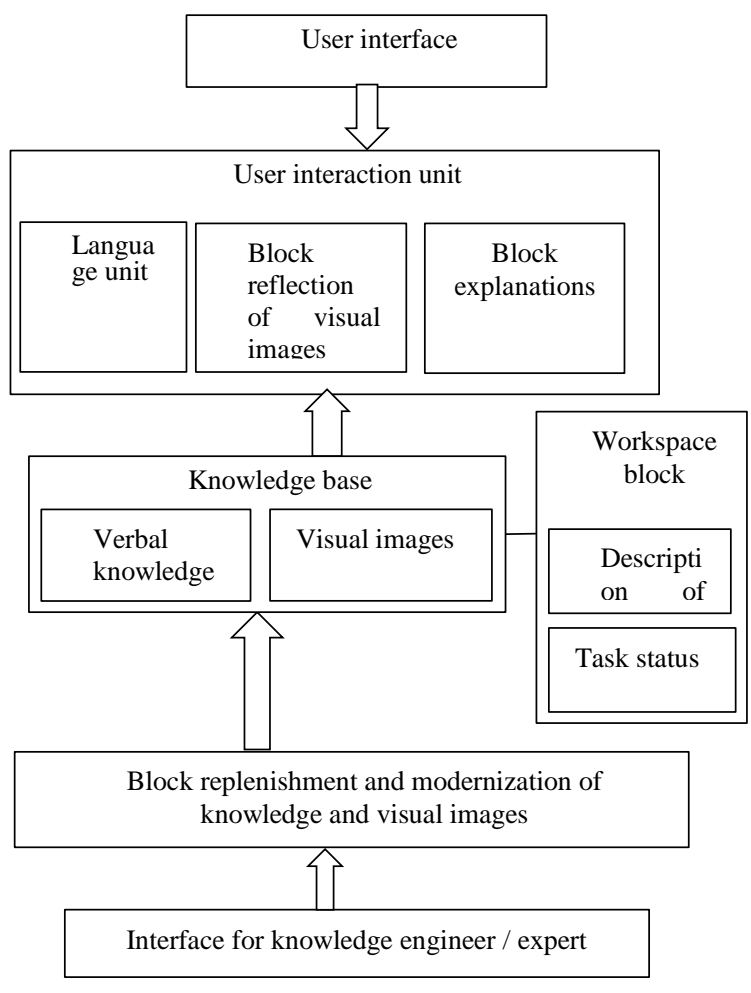

Fig. 1. The architecture of the hybrid linguistic-and-image system

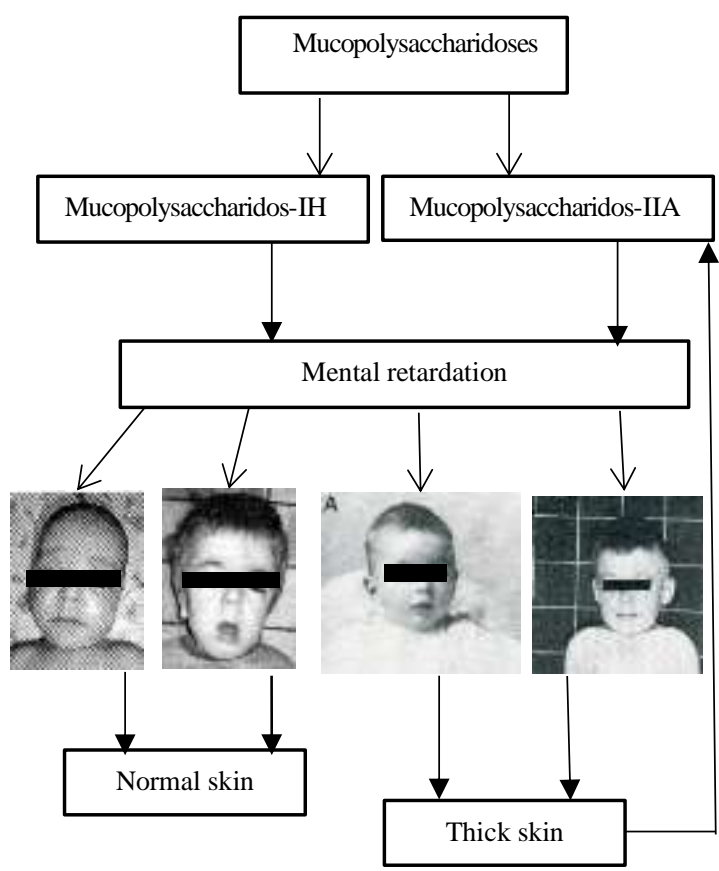

Fig.2. A fragment of the ontology of hereditary diseases (mucopolysaccharidoses)
The figure shows that the domain representing MUCOPOLYSACCHARIDOSIS class includes a number of subclasses (in this example, two of this set). Of the total number of ontologies, we see three linguistic and figurative fragments containing two rows. Linguistic ontology "mental retardation" allows experts with a high enough confidence to assume the presence of the disease one of the two subclasses (MPS-IH and MPS-II). A subsequent appeal to the imaginative ontology that allows to obtain a holistic view of the phenotypic differences in the structure of the skull and facial features of children, makes it possible to go to the related to these shaped rows linguistic ontologies, indicating a fundamentally different, antagonistic signs (skin response). On this basis, possibly to form a hypothesis about the final diagnosis.

Due to the vagueness of many medical concepts seems logical appeal to the fuzzy ontology. This is determined by the fact that the concepts and relationships, ambiguous, imprecise and do not have rigid boundaries.

Lee and colleagues [15] under the fuzzy ontology is understood as an extended ontology problem area with fuzzy concepts and fuzzy relations. The same can apply to a holistic visual representations. In addition, the construction of the ontology graph expert team, because of the possibility of having between conflicting views, even binary expert assessments of the "yes-no" result in the weighting of vertices and edges corresponding graph. Therefore, adequate means of formalizing the ontology can serve as a model on the basis of linguistic variables, fuzzy sets, fuzzy relations, fuzzy graphs. This should be attributed to the visual ontology shaped rows of objects which differ varying degrees of similarity. 
AND \{Mental retardation, Mild, Moderate $\}$

AND $\{$ Somatic symptoms, Mild $\}$

AND

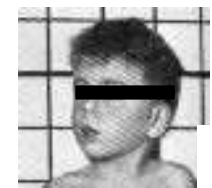

OR
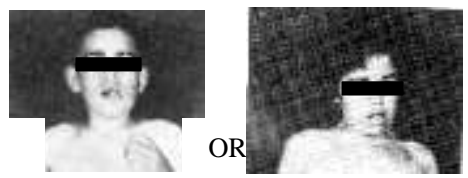

IF

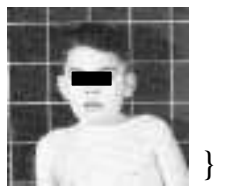

AND $\{$ hypothesis is

$\mathrm{THEN} \longrightarrow$ Confirmation

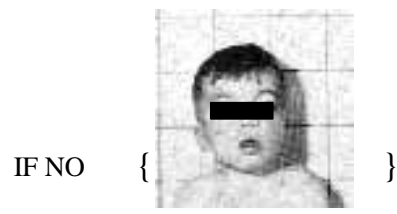

THEN $\rightarrow$ Declining

Fig. 3. Schematic representation of the hybrid frame with the decision rule

Referring to the representation of knowledge in the form of symbolic images on the frames. The frame answers the corresponding a typical member of the visual sequence (archetype, parental frame, or frame of a class), and slots are presented by diverse differing images of the given type (by "individuals") that differ in their particular nonverbalized or poorly verbalized characteristics. Visual images are contextually dependent on verbalized knowledge. The reverse situation is also possible - traditional descriptions may already be "associated" with the images that are included in a differential diagnostic series for the purpose of support a final decision. The presentation of a contextually related visual series that is kept in a knowledge base by a physician-user will make it possible to compare a new case with a well known one and simultaneously can assist one in recalling the visual images of the manifestations of a disease (association by similarity).

Visual images are included in the knowledge model for use on stages of formation and analysis of diagnostic hypotheses. Suitable image series will be presented sequentially doctors in the nomination system of diagnostic hypotheses that have not been used previously created intelligent systems. Communication between the expert system and user-physicians will be maintained at the level of specification of verbal and visual images. Cognitive function of images notion allow the physician-user will reject the erroneous diagnostic hypothesis (at the stage of their formation) and accelerate the solution of the task, including the rare atypical forms of disease. If necessary, can be performed to find additional evidence of the primary hypothesis.

An image or iconic sign that will be regarded as a symbol can in some cases serve either as an argument (additional argument) for a certain decision or as a counter argument, which results in falsifying (rejecting) an advanced hypothesis.

\section{CONCLUSION}

The traditional logical-and-linguistic intelligent systems do not ensure a unambiguous comparison between a cognitive presentation physicians and verbal description of the visual characteristics of the diseases, which have hardly characterized manifestations. This is caused by the absence of an image component in a knowledge base.

Thus, an logical-and linguistic-and-image intelligent system implies that a knowledge base contains images that reflect the characteristic properties of a patient. Their use can answer the initial stage of the procedure for the formation of a diagnostic hypothesis or be turned on in a deduction system at any step. The construction of this system can be based on the combination of arguments and/or plausible statements with a Gestalt structure in the form of visual objects of an associative image series. Diagnostic decisions will be made in this case based on logical and argumentative constructions with an image component.

Creating a new generation of logical-and-linguistic-andimage intelligent medical systems on the basis of the formal submission of hybrid knowledge and methods of inference on knowledges will improve the efficiency of information support medical diagnostics, particularly in orphan diseases. It is essential for the early start of targeted therapeutic interventions, which is especially important in progressive hereditary metabolic diseases characterized by clinical polymorphism.

\section{REFERENCES}

[1] S. Ayme, M. Caraboenf and J. Gouvernet "GENDIAG: A computer assisted syndrome identification system". Clin. Genet., vol.28, pp.410411, 1985.

[2] POSSUM. URL: http://www.possum.net.au/

[3] S.S. Guest, C.D. Evans and R.M. Winter "The online London dysmorphology database". Genet Med., vol.1, pp.207-212, 1999.

[4] B. Kobrinsky, L. Kazantseva, A. Feldman and J. Veltishchev "Computer diagnosis of hereditary childhood diseases". Med. Audit. News, vol.1, pp.52-53, 1991.

[5] A. Schieppati, J.I. Henter, E. Daiana and A. Aperia "Why rare diseases are an important medical and social issue". Lancet, № 371, pp.20392041, 2008

[6] P. Hammond, T.J. Hutton, J.E. Allanson and et al. "3D analysis of facial morphology". Am. J. Med. Genet., vol.126A, pp.339-348, 2004.

[7] P. Hammond, T.J. Hutton, J.E. Allanson and et al. "Discriminating power of localized three-dimensional facial morphology". Am. J. Hum. Genet., vol.77, pp.999-1010, 2005.

[8] S.F. Smithson and R.M. Winter "Diagnosis in dysmorphology: clues from skin”. Br. J. Dermatol., vol.151, pp.953-960, 2004.

[9] VisualDx. Logical Images Inc., http://www.visualdx.com/.

[10] E. Vardell and C. Bou-Crick "VisualDx: A Visual Diagnostic Decision Support Tool”. Med. Ref. Serv. Quarterly, vol.31, pp.414-424, 2012.

[11] Q. Ferry, J. Steinberg, C. Webber and et al. "Diagnostically relevant facial gestalt information from ordinary photos". eLife, No.3, e02020, 2014 . 
[12] K. Kuru, M. Niranjan, Y. Tunca, E. Osvank, and T. Azim "Biomedical visual data analysis to build an intelligent diagnostic decision support system in medical genetics". Artif. Intell. Med., vol. 62, pp.105-118, 2014

[13] B.A. Kobrinskii "Sequences of Images in Intelligent Systems". Sci. Tech. Inform. Proc., vol. 37, pp.328-335, 2010.

[14] B.A. Kobrinskii, "The Significance of Visual Image Presentations for Medical Intelligent Systems". Sci. Tech. Inform. Proc., vol. 40, pp.337341, 2013.

[15] Ch.-S. Lee, Zh.-W. Jian and L.-K. Huang "A Fuzzy Ontology and its Application to News Summarization". IEEE Transact. Syst., Man and Cyber., vol.35, pp.859-880, 2005. 
